\title{
La compassione di Francesco d'Assisi per gli altri e per il Creato
}

Francesco nella condivisione della sua compassione con gli altri tramite le parole e concreto l'agire rivolge il suo messaggio innanzitutto a quelli che desiderano imitare interamente il suo stile di vita che aveva ricevuto dall'Altissimo stesso, per amare Dio con tutto il cuore e con tutta la mente. Tenendo in mente quanto detto sopra, in questo articolo cercheremo di elaborare una visione globale della compassione in Francesco, presente nei suoi Scritti, la quale ci permette di capire meglio la fonte principale della sua ispirazione. L'identificazione con Dio misericordioso e compassionevole nel Cristo povero e sofferente è una costante nel pensiero del Assisinte, che appare in molteplici aspetti: prima di tutto ha creato una nuova relazione con ogni uomo, ma anche ha portato i frutti nell'amore per la natura e per le sue creature, come icone visibili del divino nella sua più immediata epifania ${ }^{1}$.

Questo articolo, nel quale ci proponiamo di parlare della compassione verso l'uomo e il Creato come è presentata negli Scritti, verrà diviso in quarto parti. Nella prima parte vedremo la visione dell'uomo nel pensiero di Francesco. Nella seconda parte prenderemo in esame l'evento dell'incontro con i lebbrosi, fondamentale per poter scoprire il fenomeno della compassione. Successivamente, nella terza parte, tratteremo la presenza della compassione verso gli uomini: i malati, i poveri, i fratel-

Cfr. Cozzaglio P., La sincronicità nel cammino spirituale e mistico di san Francesco d'Assisi, „Italia Francescana” 3 (2001), p. 34. 
li, i disprezzati e persino i nemici. Infine, nell'ultima parte, esamineremo l'atteggiamento del Santo verso il Creato.

\section{La visione dell'uomo negli Scritti di Francesco}

Per poter comprendere meglio l'atteggiamento della compassione verso gli uomini e tutto l'Universo dobbiamo prima brevemente delineare la visione dell'uomo che troviamo negli Scritti di Francesco. Le ispirazioni che hanno influito sull'immagine dell'uomo hanno avuto diversi fattori. Innanzitutto vanno sottolineate le differenti correnti intellettuali e culturali presente nell'epoca in cui visse e ammaestrasse l'Assisinte.

\subsection{I fattori e gli elementi costitutivi per la visione dell'uomo}

Nei tempi di Francesco esistevano due fondamentali concezioni nella percezione dell'uomo. La prima visione, così detta "scientifica", elaborata nei cerchi scientifici, come per esempio la scuola di Chartres, scuola cisterciense, scuola di san Vittore, ma anche creata dai alcuni pensatori indipendenti in quell'epoca. L'altra visione sarebbe quella nata nei movimenti popolari che spesso prendevano l'aspetto eretico ${ }^{2}$. Il fatto che l'uomo come tale non sia il oggetto di studio né di riflessione, costituisce una caratteristica comune di ambedue concezioni. L'uomo si presenta sempre in riferimento all'Assoluto, al suo Creatore e Redentore. Infatti, tutto pensiero del Medioevo si concentra attorno a Dio. Il punto di partenza nella riflessione sull'uomo è sempre Dio, dal momento che, la persona umana viene sempre considerata in relazione con Dio. Proprio per quel motivo san Francesco, parlando o scrivendo sull'uomo, lo percepisce sempre in questa prospettiva. Altra peculiarità di ambedue visioni dell'uomo dell'epoca, era la tendenza di ridurre la percezione dell'uomo alla sua dimensione puramente spirituale, dimenticando quella corporale. Una delle più incisive eresie d'allora, che metteva in netta opposizio-

Cfr. Gniecki C., Visione dell'uomo negli Scritti di Francesco d'Assisi, Roma 1987, pp. 34-37. 
ne lo spirito contro la materia, era quella della setta dei catari ${ }^{3}$, la quale fortemente influiva alla visione dell'uomo in quel periodo.

Francesco d'Assisi ha vissuto in un'epoca in cui l'uomo - in modo particolare ciò che in lui è corporeo - veniva spesso sottovalutato e perfino disprezzato. Conoscendo gli Scritti del Santo possiamo trovare l'ombra di questo ragionamento. In alcuni testi troviamo le espressioni in cui sembra che Francesco conoscesse l'uomo in una luce negativa, mostrandolo come decaduto soprattutto nel «corpo» come la fonte del male e dei suo vizi ${ }^{4}$. Questa considerazione dell'uomo, indica che il Santo ha avuto una visione molto concreta e reale dell'esistenza umana. L'immagine dell'uomo che traspare dagli Scritti è in linea con la visione antropologica dell'uomo che troviamo nella Bibbia $^{5}$. Analizzando gli scritti di Francesco, a prima vista si ha impressione che l'immagine dell'uomo che essi delineano è poco coerente, o addirittura, contraddittoria. Da una parte, infatti, si parla della grandezza e dignità dell'uomo, dall'altra - della sua piccolezza e viltà. Eppure, dopo un'analisi approfondita, scopriamo che non si contraddicono, perché, come vedremo dopo, si tratta di due aspetti diversi della stessa realtà umana.

\subsection{La grandezza e la dignità dell'uomo}

Negli Scritti di Francesco troveremo senz'altro tanti brani in cui Poverello si meraviglia del prodigio dell'uomo e della sua dignità che porta in sé. Senza dubbio grande influenza sulla positiva visione dell'uomo che aveva Francesco, esercitava la Sacra Scrittura, in modo particolare i Salmi e i Vangeli. La scuola cattedrale dove giovane Francesco ha imparato la liturgia e ha ascoltato la parola di Dio erano le prime fonti che lo hanno formato. L'altra fonte che ha influito sulla visione dell'uomo era la sua propria l'esperienza di vita che si manifesta nell'esperienza della

\footnotetext{
3 Ibidem, p. 24.

4 Fonti Francescane, Ammonizioni 5, 3; 10; Regola non bollata 22, 5-7; 23, 2; 23, 8; Lettera ai fedeli II 69; II 46.

5 In questa prospettiva si inserisce anche il Concilio Vaticano II nella sua Costituzione pastorale Gaudium et Spes 13, laddove dice che: «nella luce di questa Rivelazione trovano insieme la loro ragione ultima sia la sublime vocazione, sia la profonda miseria, di cui gli uomini fanno l'esperienza».
} 
sua fragilità e dell'accidentalità della sua esistenza terrena ${ }^{6}$. Il Poverello ha percepito l'uomo come un peregrino, come un essere sempre in cammino - homo viator. L'uomo, essendo pellegrino sulla terra, riconosce la presenza di Dio, la quale per Francesco si manifesta come principio e il fine della sua vita. Secondo lui, Dio ha creato tutti gli uomini, gli esseri e la natura, per la sua benevolenza, cioè per un atto di amore e di gratuità. E in questo senso Dio è fondamento dell'uomo e della natura, perché è il suo Creatore. Per Francesco è chiaro che ogni creatura umana è fatta a immagine e somiglianza di Dio ed è quindi fondamentale buona. Nel capitolo 23 della sua Regola non bollata Francesco si rivolge:

Onnipotente, altissimo, santissimo e sommo Dio, Padre santo e giusto, Signore Re del cielo e della terra, per te stesso ti rendiamo grazie, perché per la tua santa volontà e per l'unico tuo Figlio con lo Spirito Santo hai creato tutte le cose spirituali e corporali, e noi fatti a immagine tua e somiglianza? ${ }^{7}$.

L'autore in questo brano si rivolge a Dio nella persona del Padre usando gli attributi superlativi, nei quali si riconosce colui che effettivamente agisce nel mondo e nella vita dell'uomo. Quel testo contiene le importanti verità teologiche e antropologiche. La prima è quella che ol Padre è il creatore di tutto ciò che esiste, degli esseri spirituali e quelli materiali. Il Dio Padre solo è la fonte di ogni essere. Come l'opera di creazione, anche tutta l'opera di redenzione, ma pure il mistero dell'incarnazione, è l'atto amoroso di Dio Padre. Francesco, come altri autori di quel tempo, ritiene che la creazione dell'uomo sia un frutto dell'amore e della bontà divina, il dono di Dio. Per Francesco, Dio mediante la creazione agisce come Padre nella sua onnipotenza, nella sua santità e nella sua giustizia sulla creazione. L'atto della creazione è un atto della giustizia di Dio e la creazione è sottoposta alla sovranità di Dio ${ }^{8}$.

6 Su questo interessante aspetto della spiritualità del Santo d'Assisi scrive nel suo lavoro Steffan M., "Quando sono debole, è allora che sono forte» (2 Cor 12, 10). L'elogio della fragilità, „L'Italia Francescana" 82 (2007), pp. 129-139, vedi anche Maranesi P., La fragilità fonte di verità e di vita secondo Francesco di Assisi, „L'Italia Francescana” 82 (2007), pp. 105-127.

Fonti Francescane, Regola non bollata 23, 1.

8 Cfr. Freyer J. B., "Homo Viator". L'uomo alla luce della storia della salvezza. Un'antropologia teologica in prospettiva francescana, Bologna 2008, pp. 33-34. 
Accogliendo l'immagine di Gesù rivelata nei Vangeli, Francesco viene colpito da alcune specifiche caratteristiche che potremmo riassumere come «umiltà di Dio». L'Assisinte nella Quinta Ammonizione, indicando incarnazione di Figlio di Dio, sottolineava una grande dignità la quale emerge col fatto che Gesù ha preso la nostra natura umana:

Considera, o uomo, in quanta eccellenza il Signore Dio ha posto te, dal momento che ti ha creato e formato secondo il corpo e immagine del Figlio suo diletto e secondo lo spirito a sua somiglianza9 .

In questa ammonizione Francesco ha messo in evidenza uno degli aspetti fondamentali della visione dell'uomo quale è somiglianza a Cristo. L'uomo è creato ad immagine di Dio, e per essere sempre più simile a Lui deve seguire le orme di Cristo che è modello per la vita umana. In questo testo il Santo connette in modo esplicito la grandezza e la dignità della persona umana con la somiglianza divina. Ė proprio questa circostanza costituisce la grande dignità dell'uomo, il suo eccezionale stato, per il quale l'uomo stesso deve ringraziare il suo Creatore $^{10}$. Questa dignità e la grandezza dell'uomo scaturisce prima di tutto dal fatto che l'uomo è stato creato da Dio all'immagine e somiglianza del suo Figlio. Basandosi su quel testo possiamo dire che il corpo umano, cioè il visibile, fu creato all'immagine del Figlio, e lo spirito, l'invisibile, conforme alla somiglianza del Figlio di Dio. Cristo non è qui solamente l'Intermediario, attraverso il quale il Padre agisce, ma anche il Modello, all'immagine del quale il Padre aveva creato l'uomo ${ }^{11}$.

Nel capitolo 23 della Regola non bollata, Francesco esprime la sua gratitudine per il mistero dell'Incarnazione:

E ti ringraziamo, perché, come per il tuo Figlio hai creati, così per il tuo santo amore, con il quale ci hai amati, vero Dio e vero uomo dalla gloriosa sempre Vergine beatissima santa Maria lo hai fatto nascere ${ }^{12}$.

\footnotetext{
Fonti Francescane, Ammonizioni V, 1.

Cfr. Gniecki C., Visione dell'uomo..., pp. 80-85.

Ibidem, p. 83.

12 Fonti francescane, Regola non bollata 3, 3.
} 
Il frammento riportato sopra indica, che la creazione e l'incarnazione nel pensiero di Francesco, non soltanto si susseguono, ma vengono anche strettamente collegate tra di loro tramite l'amore di Dio Padre verso l'uomo ${ }^{13}$. Il testo sopra citato indica ancora una volta, che la dignità dell'uomo sorge anche dal fatto che Cristo abbia accettato il corpo umano. Come l'uomo fu creato dall'amore, nello stesso modo Dio, mosso dall'amore verso di lui, dopo la sua caduta, gli manda sulla terra suo Figlio. Francesco è cosciente che questa dignità non è soltanto la dignità dell'anima - come hanno sottolineato i teologi del suo tempo. Il Santo d'Assisi, uno dei pochi del suo tempo, indica che «al pari dell'anima, partecipa anche il corpo dell'uomo, perché, secondo Francesco, anch'esso è stato creato e formato da Dio Padre ad immagine del suo Figlio» ${ }^{14}$ lo stesso corpo di Gesù, essendo vero Uomo, doveva, nell'incarnazione, assumere anche un corpo umano con la sua natura umana.

La grandezza e la dignità dell'uomo si basano sull'amore con cui Dio ha amato. Da questo'amore derivano i doni e le qualità che costituiscono l'uomo. Secondo Francesco nell'atto della creazione l'uomo ha ricevuto le grazie e la buona volontà per seguire il Signore. Per frate Francesco è ovvio che non c'è altra pienezza dell'uomo se non la partecipazione alla vita divina perché noi «ha creato e formato secondo il corpo e immagine del Figlio suo diletto e secondo lo spirito a sua somiglianza» ${ }^{15}$. Perciò l'uomo è stato creato non perché restasse al livello umano, ma perché partecipasse alla vita divina.

\subsection{La miseria dell'uomo dopo il peccato originale}

Francesco parla anche della miseria dell'uomo. Dio, nel suo amore, aveva elargito all'uomo la libertà della quale Adamo del paradiso abusò tramite la sua disobbedienza a Dio. Attraverso l'indocilità peccatrice, l'uomo ha rovinato un legame personale con il suo Creatore, che ha portato di conseguenza l'apparizione del peccato nel mondo. Francesco

\footnotetext{
Cfr. Gniecki C., Visione dell'uomo..., pp. 88-89.

Ibidem, p. 86.

15 Fonti Francescane, Ammonizioni V, 1.
} 
è ben consapevole che lo stato attuale del creato non è quello in cui Dio lo ha posto all'origine. Egli indica evento che fu la grande svolte nella storia dell'uomo e del mondo intero quale era e il peccato di Adamo. Per quanto riguarda il peccato di Adamo, Padre Serafico descrive soprattutto le sue conseguenze: l'odio, la bruttezza, la rovina e il castigo. Nella Seconda Ammonizione ci ha dato una caratteristica del peccato:

Disse il Signore ad Adamo: "Mangia pure di qualunque albero, ma dell'albero della scienza del bene e del male non ne mangiare". Adamo poteva dunque mangiare di qualunque albero del paradiso, perché, fino a quando non contravvenne all'obbedienza, non peccò. Mangia, infatti dell'albero della scienza del bene colui che si appropria la sua volontà e si esalta per i beni che il Signore dice e opera in lui; e così, per suggestione del diavolo e per la trasgressione del comando, ne nacque il pomo della scienza del male. Bisogna perciò che ne sopporti la pena ${ }^{16}$.

Il peccato di Adamo, a somiglianza di quello degli Angeli ribelli, fu un peccato di superbia e d'orgoglio generato dalla disubbidienza poiché «colui che si appropria la sua volontà e si esalta per i beni che il Signore dice e opera in lui» ${ }^{17}$. Francesco interpreta il peccato del primo uomo alla luce della storia biblica della caduta (cfr. Gen 2, 16-17; 3, 1-3) e della dottrina di san Paolo (cfr. Rm 5, 12-19). Per lui il peccato di Adamo consiste nell'atto di disobbedienza e ribellione con la quale l'uomo tradisce il rapporto originario col Creatore.

Il testo sopra citato dice che il peccato è entrato nel mondo a causa della disobbedienza di Adamo fondata sull'«appropriazione della sua volontà». Sulla base della Seconda Ammonizione possiamo dire che l'uomo commette il peccato ogni volta che s'appropria o s'attribuisce di ciò che sta facendo di proprie forze, e inoltre, quando si vanta del bene fatto da se stesso, ritenendosi padrone di se stesso e rifiutando la dipendenza dal Dio Creatore. Per Francesco è chiaro che il peccato ha origine nella libera responsabilità. Ma causa del peccato non è il corpo. Francesco considera corpo nel senso paolino come realtà carnale che significa l'uomo vecchio, al quale si oppone lo Spirito e la vita secondo lo spirito. Il peccato ha origine nell'interno, nel centro della persona, nel-

16 Fonti Francescane, Ammonizioni II, 1-5.

17 Fonti Francescane, Ammonizioni II, 3. 
la sua volontà. L'uomo usando male il dono la libertà rifiuta la ricerca di Dio e l'orientamento verso Dio, che è il Sommo Bene stesso. Con questo nella sua ostinazione si fa misura del mondo. L'uomo rifiuta «l'atteggiamento di gratitudine che spetta a Dio e quindi nega la vita come dono. Si fa autore della vita e quindi come estrema conseguenza padrone della vita e della morte» ${ }^{18}$.

Per mezzo del primo uomo, il peccato e il male entrarono in questo mondo e lo dominarono. La conseguenza della disobbedienza di Adamo coinvolge tutta l'umanità e ciascun uomo. In quest'ottica si comprende affermazione di san Francesco secondo la quale ogni uomo partecipa nel causare la morte di Dio:

E tutte le creature, che sono sotto il cielo, per parte loro servono, conoscono e obbediscono al loro Creatore meglio di te. E neppure i demoni lo crocifissero, ma tu insieme con loro lo hai crocifisso, e ancora lo crocifiggi quando ti diletti nei vizi e nei peccati ${ }^{19}$.

Ogni uomo quando pecca e persevera con predilezione nel peccato, è personalmente responsabile della morte di Gesù Cristo. Finché l'uomo rimane nel peccato causa nuovamente la crocifissione di Cristo.

\subsection{La redenzione e la salvezza dell'uomo}

L'uomo trova la sua piena comprensione alla luce del mistero di Dio. All'inizio del Commento al Padre nostro Francesco insegna: «O santissimo Padre nostro: creatore, redentore, consolatore e salvatore nostro» ${ }^{20}$ Quel frammento rivela il fatto che il Dio in Francesco non è solo Creatore, ma anche Redentore, Consolatore e Salvatore dell'uomo. Malgrado l'errore dell'uomo, Dio non ha smesso di amarlo né finito di prender cura del suo destino. Dopo il peccato del primo uomo Dio ha annunciato il suo piano di salvezza, per mezzo di cui ha rinnovato la relazione perduta e ha ristabilito la dignità rovinata. San Francesco ne scrive nella Regola non Bollata:

\footnotetext{
18 Freyer J. B., “Homo Viator"..., p. 228.

19 Fonti Francescane, Ammonizioni 5, 3.

20 Fonti Francescane, Preghiera sul Padre Nostro 1.
} 
E noi per nostra colpa siamo caduti. E ti ringraziamo, perché, come per il tuo Figlio ci hai creati, così per il tuo santo amore, vero Dio e vero uomo dalla gloriosa sempre Vergine beatissima santa Maria lo hai fatto nascere e per la croce e il sangue e la morte sua hai voluto liberare noi prigionieri ${ }^{21}$.

Francesco nella contemplazione dell'amore di Dio Padre rivelatasi nel fatto dell'incarnazione del suo Figlio Prediletto ha scoperto in Lui «vero Dio e vero uomo dalla gloriosa sempre Vergine beatissima santa Maria lo hai fatto nascere ${ }^{22}$ che per la sua Passione e Morte ha redento l'uomo. La Redenzione svela definitivamente una verità assoluta su Dio. Il Figlio di Dio, abbracciando la forma umile del Servitore di Yahve e del servo dell'uomo, si fa il sacramento dell'amore umile di Dio ${ }^{23}$. Di quell'esperienza che visse il Santo di Assisi, sorge «l'immagine di Dio quale amore umile, povero, attivo. Amore che scese fino al libello della umile e povera esistenza dell'uomo» ${ }^{24}$.

Francesco osserva che al dramma in cui l'umanità era travolta, Dio rispose con la grazia e la misericordia. Il Creatore non ha abbandonato l'uomo nella sua caduta, ma come per amore lo aveva creato, così mosso dallo stesso amore, avendo compassione dell'uomo che si era allontanato di Lui, ha mandato il suo Figlio per liberarlo «da ogni male» permettendogli di vivere in comunione con Lui. Secondo Francesco l'incarnazione e la croce di Gesù è la manifestazione dell'amore salvifico di Dio. In quest'atto di amore la croce diventa uno strumento di gloria per Cristo e di riparazione ed espiazione del peccato dell'uomo e il fattore che restituisce la libertà all'uomo.

Riassumendo possiamo osservare che la visione dell'uomo quale troviamo negli Scritti di Francesco non è astratta, non è frutto di ragionamenti filosofici, ma è il frutto dell'esperienza della vita quotidiana. Secondo Francesco, che decifra la realtà attraverso la Bibbia, tutto è stato creato da Dio ed è il frutto dell'amore divino. Tutti gli esseri, quelli spirituali come

21 Fonti Francescane, Regola non bollata 23, 2-3.

22 Fonti Francescane, Regola non bollata 23, 3.

${ }_{23}$ Cfr. Synowczyk K., Miłość w nauczaniu św. Franciszka z Asyżu, „Studia Franciszkańskie” 17 (2007), p. 59.

24 Baran B., Franciszkańska droga do zjednoczenia z Bogiem, „Studia Franciszkańskie” 11 (2001), p. 37. 
quelli materiali, sono l'opera della bontà e dell'amore di Dio Padre, tra i quali l'uomo occupa un posto particolare. Francesco percepisce l'uomo come la creatura che rispecchia le opere di Dio, che segue Cristo per essere in comunione con Dio. La fonte di questa visione la troviamo innanzitutto nella Sacra Scrittura. L'uomo nella sua prospettiva è visto nella sua unità corporeo - spirituale: l'uomo tutto intero (anima e corpo) è creato ad immagine e somiglianza di Dio, e non solo anima. Secondo Francesco d'Assisi la dignità dell'uomo scaturisce non solo dall'anima ma anche del corpo ${ }^{25}$.

Non solo l'anima, ma anche il corpo umano e tutto l'ambito corporale dell'uomo è vista sotto l'aspetto positivo. La dimensione corporale dell'essere umano è mostrato come condizione della presenza in mezzo al mondo creato da Dio. Il corpo permette all'uomo il contatto con il prossimo e con altre creature. Nella realtà materiale, nel mondo che lo circonda, contemplato con gli occhi di fede, Francesco scopre il Creatore e la strada verso di Lui. Questa prospettiva sarà sviluppata più avanti laddove analizzeremo il Cantico delle Creature. La dimensione corporale, essendo il modo necessario di entrare nelle relazioni con il creato, presenta anche la maniera di stabilire il rapporto con Cristo presente nel mondo e di vivere in unione con Lui.

\section{L' incontro con i lebbrosi:}

il volto dell'uomo sofferente rivela quello di Dio

Prima di parlare della compassione nei rapporti con i fratelli e gli uomini bisognosi in genere negli Scritti del Poverello, desideriamo presentare un evento della vita di Francesco - importante per la nostro ricerca - che ha segnato indelebilmente la sua vita e il suo atteggiamento verso il prossimo. Questo evento è descritto nel passo che si trova nel Testamento di frate Francesco. Breve, ma preziosissimo racconto autobiografico della sua conversione è fondamentale per la comprensione dell'esperienza della compassione in Francesco.

25 Cf. Zavalloni R., L’uomo e il suo destino nel pensiero francescano, Porziuncola, Assisi, 1994; Freyer J., "Homo Viator". 
La singolarità di quel documento sta nel fatto che esso in un modo speciale ci avvicina alla conoscenza degli elementi più fondamentali della sua spiritualità, che sono effetto della sua adesione al modello di vita secondo il Vangelo. In esso vengono ribaditi i principi fondamentali della sua forma di vita ${ }^{26}$. Il Testamento, nato negli ultimi giorni della vita di Francesco, costituisce una voce del padre preoccupato, che desidera che i suoi figli spirituali vivano secondo la Regola nel modo più perfetto. Il documento nacque nelle circostanze particolari, mentre la fraternità era agitata a causa del conflitto riguardante l'attuazione degli ideali del Poverello nella vita concreta delle prime fraternità.

Il Testamento, essendo il documento personale di Francesco, costituisce senza dubbio la chiave di volta per la piena comprensione degli ideali che Francesco ha incarnato nella sua vita. Il Testamento è l'ultima opera del Serafico Padre che è stata scritta, tra apprie a settembre del 1226, quando Francesco ha lasciato il vescovado di Assisi e si è trasferito alla Porziuncola. Il Testamento ha carattere del "discorso d'addio" similmente come i testamenti spirituali che troviamo nella Bibbia ad esempio: Giacobbe, Mosé e ultimi momenti di vita di Gesù Cristo descritti nel Vangelo di Giovanni ${ }^{27}$.

Prima della sua morte Francesco ha scritto:

Il Signore così diede a me, fratello Francesco, di iniziare a fare penitenza, poiché, essendo nei peccati, troppo mi sembrava amaro vedere i lebbrosi. E lo stesso Signore mi condusse in mezzo a loro e feci misericordia con loro. E allontanandomi da loro, ciò che mi sembrava amaro mi si trasformò in dolcezza d'animo e di corpo. E poi un poco ristetti e uscii dal secolo ${ }^{28}$.

Con queste parole malato e moribondo frate Francesco comincia il suo ultimo scritto - il Testamento. L'incontro con il lebbroso ${ }^{29}$, da che co-

${ }^{26}$ Cfr. Merlo G. G., Testamento, [in:] Francesco D’Assisi, Scritti. Testo latino e traduzione italiana, Padova 2002, p. 429.

${ }_{27}$ Cfr. Sykuła H., Testament św. Franciszka i św. Klary. Studium teologiczno-duchowe, Kraków 2010.

28 Fonti Francescane, Testamento 1-3.

${ }_{29}$ I lebbrosi nella società medievale risiedevano in così detti lebbrosari, situati fuori dei luoghi abitati dalla gente sana. L'apogeo della malattia della lebbre avviene nei secoli XII e XIII. In quel tempo sorgono anche molti lebbrosari. Ai lebbrosi erano imposte molte restrizioni - tra le altre, non potevano uscire fuori del lebbrosario e circolare per le strade, purché si servano del bat- 
mincia le sue rievocazioni, e il rifiuto della vita di un giovane mercante e il re della gioventù d'Assisi, fatta fin a quel momento, costituisce il contenuto più personale del testo. In esso Francesco ricordava i lebbrosi, che prima gli ispiravano i sentimenti di ripugnanza. «E il Signore stesso mi condusse tra loro e usai con essi misericordia» ${ }^{30}$. Dopo questo evento per giovane Francesco i lebbrosi si presentano come suoi maestri, come coloro che gli hanno comunicato un gusto nuovo della «dolcezza» della vita stessa ${ }^{31}$.

E interessante notare che Francesco in punto di morte, tra tanti eventi della sua vita, che noi possiamo conoscere grazie alle sue biografie, abbia riconosciuto come una "svolta" principale della sua vita proprio l'incontro con i lebbrosi. L'importanza di questo evento sottolinea Cesare Vaiani scrivendo:

Non verrà sottolineata mai abbastanza l'importanza di questo riferimento ai lebbrosi per il successivo sviluppo dell'esperienza di Francesco: è lui a dire come il proprio cambiamento di vita non sia legato alla preghiera, o ai sogni che gli indicano la volontà del Signore, ma principalmente all'incontro con i lebbrosi. Poi, nel ricordo del Testamento, verrà anche la preghiera nelle chiese e il riferimento alla croce: ma dopo i lebbrosi, che restano il primum della sua conversiones ${ }^{32}$.

Senza dubbio, ci furono anche altri segni e chiamate del Signore, ma Francesco nel servizio ai lebbrosi, nell' «usare misericordia», considerava il momento decisivo del proprio cambiamento autentico della vita e del modo di pensare. Dobbiamo sottolineare che Francesco dettando il Testamento alla fine della sua vita, vede l'iniziativa di Dio nel fatto dell'incontrato con i lebbrosi affermando: «il Signore stesso mi condusse tra loro». Il Poverello è consapevole del fatto che il suo avvicinamento ai lebbrosi, come anche la sua conversione, erano la grazia di Dio. L'incontro con i lebbrosi fu l'evento decisivo che lo induse a prendere la

\footnotetext{
tola, facendo chiasso. Nel 1197 il Concilio Lateranense ha permesso la costruzione delle cappelle e la fondazione dei cimiteri per i lebbrosi. Cfr. Goff J., Kultura średniowiecznej Europy, Warszawa 1995, p. 322; Maranesi P., L'eredità di frate Francesco. Lettura storico-critica del Testamento, Assisi 2009.

Tommaso da Celano, Vita del beato Francesco (Vita prima) 17.

Bartoli M., Pater pauperum..., p. 35.

32 Vaiani C., Io sto coi lebbrosi, [in:] Dozzi D. (a cura di), Il Vangelo della misericordia, Bologna 2006, pp. 15-16.
} 
decisione di cambiare la sua vita ${ }^{33}$. Tommaso da Celano, nella Vita beati Francisci, ricordando l'incontro con il lebbroso, ha descritto Francesco, che alla vista del malato, inizialmente aveva voglia di scappare, ma avendo superato se stesso, "gli si avvicinò e lo baciò»" ${ }^{34}$. Sotto l'espressione "usai con essi misericordia», si nascondono l'insieme dell'atteggiamento del Poverello verso gli ammalati della lebbra: il bacio, il servizio, la compassione, l'elemosina.

In questo l'incontro il giovane Francesco ha capito che soltanto la misericordia assieme alla compassione rende reale il nostra amore nei riguardi degli altri, e nello stesso tempo, si rende conto che è nell'incontro con i lebbrosi che la concezione dell'Incarnazione e della sequela del Cristo povero e crocifisso raggiunge una nuova e impegnativa forma. I lebbrosi per Francesco sono stati come uno specchio, attraverso il quale egli può scoprire, con la vittoria sul proprio egoismo, la decisiva vittoria della Grazia divina, fino ad arrivare a vivere la misericordia e la compassione del Vangelo (cfr. Lc 10, 25-37), giacché come Francesco ci spiega nel Testamento: «essendo nei peccati, troppo mi sembrava amaro vedere i lebbrosi ${ }^{35}$, era il peccato quello che impediva di riconoscere nei lebbrosi l'immagine del Dio sofferente.

La conversione del Poverello, avvenuta grazie all'incontro con i lebbrosi, che si è manifestata concretamente nel cambiamento di percezione dei valori: amaro - dolce, gli ha aperto gli occhi alla sofferenza umana universale, la quale abbraccia sia l'anima (la lebbra dell'anima) e il corpo. Il Santo ha visto in questi ammalati, indigenti uomini, il mistero di Gesù Cristo, che pur essendo Dio, per amore dell'uomo si è degnato "scendere", "abbassarsi", "diventare la piccolezza", "umiltà", "povertà", per creare un vero e proprio legame dell'unità di vita con l'uomo ${ }^{36}$. Dal profondo di quel'esperienza fondamentale è nato in Francesco il desiderio di seguire quell'amore umile e povero del suo Signore. L'amore

\footnotetext{
33 Ibidem, p. 36.

34 Fonti Francescane, Testamento 2.

35 Fonti Francescane, Testamento 1.

36 Cfr. Immarrone G., Duchowość franciszkańska. Istota i podstawowe treści. Chrześcijańska propozycja życia na dziś, Kraków 1998, p. 51.
} 
che prendeva forma di spogliarsi, di una rinuncia totale a tutto, nella povertà, umiltà e piccolezza.

Nel incontro con il lebbroso Francesco tocca i radici della sua umana esistenza e entra sulla via che conduce alla vita. Nel lebbroso non incontra soltanto un uomo sofferente ma innanzitutto incontra sofferente Figlio dell'uomo (Is 53,4). Il suo sguardo cambia. Egli non vede più il lebbroso. Lo ha baciato $\mathrm{c}$ non lo vede più. Così interpreta il Celano. Infatti non lo vede più come lebbroso. Subito dopo. sempre condotto dallo Spirito. scopre il Crocifisso. «Da quel momento si fissò nella sua anima la compassione del Crocifisso» ${ }^{37}$.

Nell'evento dell'incontro con i lebbrosi, Francesco li mostra in particolar modo la misericordia. In questa prospettiva è interessante accostare l'atteggiamento del buon Samaritano della omonima parabola e quello di Francesco che incontra i lebbrosi. Il buon samaritano accorre in aiuto al prossimo in bisogno mosso dalla compassione, mentre Francesco si accosta ai lebbrosi sotto ispirazione proveniente dal Signore. La parola misericordia ci ricorda la parabola del Buon Samaritano (Lc 10, 25-37).

L'atteggiamento della compassione del Padre Serafico è una conseguenza della sua esperienza di Cristo - povero e umile. Francesco prima ha scoperto dei lebrosi (poveri, sofferenti e rifiutati), e dopo questa esperienza - ha scoperto il Cristo povero. Il Poverello percepisce un legame molto stretto tra l'atteggiamento di povertà e d'amore, che genera quello della compassione. La compassione vista sotto questa luce, appare come conseguenza dell'amore che si offre, e diventa un mistero della presenza di Gesù Cristo nell'uomo povero nel senso largo; Cristo che si rivela in ciascuno che si rende simile a Lui ${ }^{38}$. Nel modo di capire le cose di san Francesco questo atteggiamento interiore è espresso perfettamente nella parola minoritas, che indica contemporaneamente la povertà e l'umiltà evangelica, semplicità, atteggiamento di compassione e servizio ai bisognosi ${ }^{39}$. Essere "minore" non significava per il Santo

37 Tommaso da Celano, Memoriale nel desiderio dell'anima (Vita seconda) 10.

38 Cfr. Iriarte L., Vocazione francescana. Sintesi degli ideali di san Francesco e di santa Chiara, Bologna 2006, p. 157.

${ }_{39}$ Cfr. Lipiński H. J., Duchowość franciszkańska, [in:] Bejze B. (a cura di), W nurcie zagadnień posoborowych, vol. 14, Warszawa 1981, p. 388. 
far parte di una classe sociale (minores - maiores), ma il modo di vivere secondo il modo di vivere di Cristo ${ }^{40}$. E proprio per quel motivo voleva imitare il suo Signore nella sua umiltà e l'amore per ogni uomo nel suo atteggiamento di servizio.

Il Testamento menziona, che il Poverello fin dall'inizio del suo cambiamento interiore (metanoia), mosso dalla compassione, aiutava la gente più povera: "e il Signore stesso mi condusse tra loro e usai con essi misericordia" ${ }_{41}$. Sotto l'espressione "usare misericordia" si debba intendere ogni gesto, ogni azione di frate Francesco che dava sollievo e portava speranza a quella povera gente. Il Testamento ci fa vedere un fatto molto importante: Francesco non solo aiutava i bisognosi all'immagine del evangelico Samaritano misericordioso, ma anche ha accettato liberamente il loro modo di vivere, è diventato uno di loro: "e il Signore stesso mi condusse tra loro"42. Il Poverello non esitava spogliarsi del suo onore e maestà, per abbassarsi fino al livello dell'uomo che nel mondo medievale non contava nulla, viveva fuori dei margini della società. Il fondamento di una tale decisione fu la sua basilare esperienza spirituale: un incontro personale con Cristo umile e povero, che per Frate Francesco è divenuto il modello dell'amore per ogni uomo.

\section{La compassione per gli altri}

L'esperienza dell'incontro che ha avuto giovane Francesco con i lebbrosi, la quale abbiamo esaminato nel paragrafo precedente, ci ha fatto scoprire che l'amore manifestato nella compassione, e il servizio verso i lebbrosi, era per lui un dato fondamentale per costruire una autentica fraternità e solidarietà con le persone segnate dalla sofferenza di ogni genere. Poverello, spinto dall'esperienza dell'incontro con Dio, che gli apparve nell'uomo sofferente, portava in sé un profondo desiderio di servire le persone più bisognose. Il frutto di quell'esperienza divenne l'esor-

\footnotetext{
$40 \quad$ Cfr. Iriarte L., Vocazione francescana..., p. 177.

41 Fonti Francescane, Testamento 2.

42 Ibidem.
} 
tazione di manifestare attivamente gli atti di compassione alle persone che si trovavano ai margini della società di allora. Nella parte presente della nostra ricerca, l'oggetto dell'analisi costituiranno i frammenti dei diversi Scritti di Francesco; in primis: la parte principale del Commento al Padre nostro, poi, i frammenti della Regola non bollata, e in fine, i frammenti delle Ammonizioni.

\subsection{La compassione per i malati e i poveri}

Come punto di partenza nella nostra ricerca della comprensione del nocciolo della questione della compassione di Francesco verso i prossimo, prenderemo il frammento del Commento al Padre nostro, chiamato anche Esposizione o Parafrasi del Padre nostro. E' un testo senza dubbio autentico, perché si trova nei codici a partire dal XIV secolo. Invece, la sua originalità può suscitare dei dubbi, giacché comunemente si avanza l'ipotesi che san Francesco si abbia potuto servire dei commenti già esistenti a proposito di quella preghiera, imprimendo loro un carattere personale, che possiamo paragonare con altre sue opere ${ }^{43}$. La preghiera del Padre nostro, nella versione abituale di Mt 6, 9-13, risulta composta da una invocazione iniziale, ampliata mediante una frase relativa, e da sette richieste. Nel suo commento fatto preghiera, Francesco si porta innanzitutto all'invocazione iniziale e alla sua esplicitazione «che sei nei cieli», e poi alle sette richieste, dividendo però la quinta richiesta in due parti ${ }^{44}$.

Il frammento qui sotto riportato costituisce il commento alla quinta richiesta della preghiera:

Sia fatta la tua volontà come in cielo così in terra: affinché ti amiamo con tutto il cuore pensando sempre a te, con tutta l'anima desiderando sempre te, con tutta la mente dirigendo a te tutte le nostre intenzioni, cercando il tuo onore in tutto, e con tutte le nostre forze spendendo tutte le nostre forze e i sentimenti dell'anima e del corpo in ossequio del tuo amore e non in altro; e amiamo il nostro prossimo come noi stessi attirando tutti al tuo amore, secondo le nostre forze, godendo dei beni altrui come dei nostri e soffrendo con loro dei mali e senza portare alcuna offesa a nessuno ${ }^{45}$.

43 Cfr. Vaiani C., Preghiera sul Padre Nostro, [in:] Francesco D’Assisi, Scritti..., pp. 135-136.

44 Lehmann L., Francesco maestro di preghiera..., p. 195.

45 Fonti Francescane, Preghiera sul Padre Nostro 5. 
Le parole sopraindicate, essendo il più ampio commento della richiesta analizzata, prendono il posto centrale nel Commento al Padre nostro. In questa invocazione Francesco spiega in modo molto approfondito, in che cosa consiste la volontà di Dio: essa è l'amore, l'amore di Dio e del prossimo. I pensieri molto profondi del commento debbono essere considerati come frutto di una meditazione prolungata del Padre nostro. Alcune espressioni indicano un'alta teologia, o meglio una profonda conoscenza ed esperienza di Dio. Il testo è un esempio di meditazione pregata della sacra Scrittura, il che significa che la meditazione del testo biblico, quello del Padre nostro, consiste in un ampliamento delle singole espressioni, spesso compiuto con il riscorso ad altre espressioni bibliche, secondo il principio per cui la Scrittura illustra la Scrittura ${ }^{46}$. Nel nostro testo, un esempio è offerto dall'ampliamento dell'invocazione «sia fatta la tua volontà» dove san Francesco identifica la volontà di Dio attraverso il testo evangelico sul primo comandamento, che precisa l'amore di Dio e del prossimo. La fusione dei due testi biblici sulla base del comune denominatore della volontà e comandamento di Dio, spiega le parole di Francesco.

Una tale veduta del autore porta al pieno rivolgersi verso Dio nella prontezza assoluta di servire un altro. Alla luce del commento a questa richiesta, possiamo capire come dobbiamo amare i nostri prossimi. Il punto di partenza per Francesco costituisce la disposizione per attirare con ogni nostro potere tutti gli uomini all'amore di Dio. Successivamente, il Poverello vede la necessità di una schiettezza nello spirito di libertà per poter rallegrarsi del bene degli altri come del proprio bene. Finalmente, il Padre Serafico sollecita che l'uomo, ispirato dall'amore di Dio stesso compartecipi nel soffrire con gli altri per le loro sventure e dolori «godendo dei beni altrui come dei nostri e soffrendo con loro dei mali». Detto in modo molto generale, l'amore del prossimo consiste innanzitutto nel condurre, per quello che possiamo, tutti per poter scoprire l'amore di $\mathrm{Dio}^{47}$. Quest'amore verso il prossimo diventa autentico quando il bene degli altri si fa per noi una gioia tale come se fosse il nostro. Questo fe-

46 Cfr. Vaiani C., Preghiera sul Padre..., p. 137.

${ }_{47}$ Cfr. Lehmann L., Francesco maestro di preghiera..., pp. 204-205. 
nomeno del condividere i diversi aspetti della vita che porta alla compassione afferma giustamente Leonard Lehmann:

Riconoscere agli altri le loro capacità, lodarli e gioire con loro, è per Francesco la forma quotidiana dell'amore. Una gioia condivisa è una gioia doppia. Anche la compassione appartiene alla quotidianità dell'amore del prossimo. Una sofferenza condivisa è una sofferenza dimezzata. Dove si condivide gioia e dolore, lì si sperimenta quella "societas beata", quella comunione beata e beatificante di cui prima parlava Francesco. In una tale comunione, in un tale rapporto si fa esperienza dell'amore di Dio, poiché vi è la condivisione dei beni sia materiali che spirituali, e la gioia di tale compartecipazione. Da tutto ciò si sprigionano delle forze nuove che permettono di lavorare efficacemente per il regno di Dio ${ }^{48}$.

Un'ulteriore continuazione del pensiero di Francesco, confermando l'atteggiamento della compassione che lo caratterizzava di fronte alle persone bisognose, è il frammento proveniente dal nono capitolo della Regola non bollata. Questo documento costituisce un esempio dello scritto di carattere normativo. E' una testimonianza del francescanesimo primitivo, che era diventato il principio di vita per i compagni del Padre fondatore dell'ordine. Il frammento della Regola citato qui sotto incita all'imitare di Gesù Cristo nel mistero del suo avvilimento. L'accettazione dello stesso atteggiamento che caratterizzava Gesù permette - secondo frate Francesco - di godere della libertà e di gioia nati dal fatto di una vicinanza verso gli uomini sofferenti e respinti. Il Santo d'Assisi esorta i suoi frati:

Tutti i fratelli cerchino di seguire l'umiltà e la povertà del Signore nostro Gesù Cristo e si ricordino che non ci occorre avere nient'altro da tutto il mondo, se non, come dice l'apostolo, di avere il cibo e di che coprirci e siamo contenti di questi. E devono gioire, quando vivono tra persone di poco conto e disprezzate, tra poveri e deboli, infermi e lebbrosi e mendicanti per la strada ${ }^{49}$.

Il frammento della Regola qui sopra riportato mette una luce nova, mostrando come si deve comportare con i prossimi che sono in un biso-

${ }^{48} \quad$ Ibidem, p. 206.

49 Fonti Francescane, Regola non bollata IX, 1-2. 
gno particolare: «persone di poco conto e disprezzate, poveri e deboli, infermi e lebbrosi e mendicanti per la strada». Loro dovrebbero diventare "oggetto" di una cura particolare alla quale i frati sono invitati verso gli uomini che incontrano. Francesco, farsi suo il punto di vista di un grande Santo dell'antichità cristiana, un romano, Lorenzo diacono e martire, intravede in loro effettivamente degli autentici "tesori della Chiesa". La loro mala sorte, le sofferenze e diseredazione ispira a Francesco un profondo desiderio di compatire che porterà a essere presente in mezzo a loro e a condividere con loro la loro sorte. Un tale modo di guardare del Poverello sull'emarginati era condizionato dal fatto che il Santo stesso abbia sofferto molte avversità della vita e infermità nel corpo. Francesco, anche attraverso la malattia e i nel incontro con i malati più ripugnanti della sua epoca, ha scoperto l'amore di Cristo.

Il successivo frammento fondamentale che ci permetterà di scoprire in modo più profondo l'atteggiamento della compassione verso il prossimo, proviene da una raccolta di ventotto testi, più o meno brevi, che tradizionalmente vengono denominati Ammonizioni. Questi sono i consigli e insegnamenti tenuti da Francesco durante i capitoli, raccolti in seguito e presentati in forma scritta da uno dei segretari del Padre Serafico. Il titolo dell'Ammonizione Diciottesima dice: La compassione verso il prossimo. Questa ammonizione è l'unico luogo negli Scritti del Santo dove incontriamo la parola "compassione". Ciò nonostante, secondo l'unanime opinione degli esperti degli Scritti del Santo, il titolo è stato aggiunto nel periodo ulteriore dal copista allo scopo di ordinare e definire l'argomento dell'ammonizione. In quel frammento Francesco insegna:

Beato l'uomo che sostiene il prossimo suo secondo la sua fragilità in ciò che da esso vorrebbe essere sostenuto, se si trovasse in una situazione simile. Beato il servo che restituisce ogni suo bene al Signore Dio, poiché colui che avrà tenuto per sé qualcosa, nasconde in sé il denaro del Signore Dio suo e, quanto pensava di avere, gli verrà tolto ${ }^{50}$.

Francesco utilizza la parola fragilità in riferimento al prossimo. Essa vuole dire: caducità, fragilità, debolezza, futilità. In questa prospettiva il Santo vuole accogliere il prossimo in quanto tale, così come gli si ri-

50 Fonti Francescane, Ammonizioni XVIII 1-2. 
vela. Accettare il prossimo non solo con la sua ricchezza dell'intelletto, della personalità, ma anche con tutto ciò che è in lui di debole, fragile, esile e irritante. Sopportare il suo prossimo con un vasto spettro dei suoi difetti e insufficienze indica la capacità di superare se stesso per poter accendere l'amore dentro di sé e offrire al prossimo la compassione che ne deriva ${ }^{51}$.

Secondo Francesco, è necessario sopportare il prossimo così come si vorrebbe essere sopportato nelle condizioni simili. Il Santo, cosciente che ogni uomo è segnato dal marchio del peccato originale, che dopo il peccato del primo uomo era colpito tutto il genere umano, vuole capire e accogliere l'altro tramite lo svelare la verità su se stesso davanti a Dio, alla luce della Bibbia. Malgrado la debolezza in lui presente, il prossimo incontrato si rivela sempre come un dono di Dio, come uno che possiede in sé una dignità imparagonabile di figlio di Dio.

\subsection{La compassione verso i fratelli - "amore materno"}

Il frate Francesco ardeva d'un grande amore verso Dio e da questo amore nasceva l'atteggiamento della compassione verso il prossimo e, in modo particolare, verso i suoi confratelli. Avendo scoperto l'amore divino nel Cristo povero, crocifisso e modesto, rispondeva con un simile amore a Lui e ai fratelli. L'esperienza dell'amore di Dio vissuta da Francesco e la vocazione comune con i confratelli, hanno formato due fonti principali del suo amore compassionevole verso i frati a lui affidati. «Eravamo illetterati e sottomessi a tutti ${ }^{52}$. Così scrive frate Francesco nel Testamento, ricordando gli inizi della fraternità. Nella minorità esprime la sua relazione con l'altro. «Tutti siamo chiamati semplicemente frati minori», scrive nella Regola non bollata, e subito aggiunge a commento le parole del Vangelo: «e l'uno lavi i piedi all'altro» (Gv 13,14). La minorità parla attraverso l'altro, attraverso il servizio e il prendersi cura che crea la vicinanza e ci rivela fratelli. La minorità è dunque via al prossimo e nell'essere fratelli ci si scopre tutti minori. L'una non è possibile senza l'altra.

51 Cfr. Messa P., Profili L., Il Cantico della fraternità: le Ammonizioni di Francesco d'Assisi, Assisi 2003.

52 Fonti Francescane, Testamento 19. 
Francesco lo sottolinea specialmente nel Testamento: «E dopo che il Signore mi diede dei fratelli, nessuno mi indicava che cosa dovessi fare; ma lo stesso Altissimo mi mostrò che dovevo vivere secondo il modello del santo vangelo» ${ }^{53}$. Dunque, la vocazione comune rappresentava per il Santo la sorgente di un amore tenero e premuroso verso i confratelli. Francesco, avendo la consapevolezza del fatto che i frati siano un dono di Dio, voleva che loro formassero una comunità su modello della famiglia. La vocazione comune doveva unire i frati in tal modo che si formassero i legami di una vera e piena di amore famiglia umana:

E ovunque sono e si troveranno i fratelli, si mostrino familiari a vicenda tra di loro. E senza timore ognuno manifesti all'altro le proprie necessità, perché, se la madre nutre ed ama il proprio figlio carnale, con quanto più amore deve uno amare e nutrire il suo fratello spirituale ${ }^{54}$

Francesco, paragonando l'amore tra i frati all'amore tra la madre e il figlio, abbia dovuto probabilmente da solo sperimentare la gioia dell'amore materno. Grazie a questa esperienza, possedeva un modello dall'amore vero, premuroso e tenero, al quale poteva riferire l'amore fraterno. Ma nell'atteggiamento di Francesco possiamo scorgere qualcosa in più. Nella famiglia minoritica deve predominare un amore ancora più tenero e più servizievole, un amore ancora più intenso da quello della madre verso il suo figlio. I frati lasciano il seno della famiglia naturale e cominciano la vita nella nuova famiglia, la quale assume in tal modo la condizione soprannaturale. Per formare una tale comunità occorre anche un amore maggiore di quel naturale - un amore trascendente, che solo Dio concede.

La lettera personale del Santo a frate Leone costituisce una testimonianza concreta del suo tenero amore ${ }^{55}$. Anche se il suo contenuto sembra poco chiaro - Francesco scrive delle questioni conosciuti soltanto da

53 Fonti Francescane, Testamento 14.

54 Fonti Francescane, Regola bollata 6, 7-8; cfr. Fonti Francescane, Regola non bollata 9, 10-11.

55 Frate Leone era uno dei primi frati di san Francesco. Più tardi è diventato il suo particolarmente caro compagno, apparteneva al gruppo dei pochi che lo assistevano nel ultimo periodo della vita. Alcuni sostengono che erano uniti con un legame di amicizia. Cfr. Manselli R., Święty Franciszek..., pp. 424-425. 
lui stesso e da frate Leone - tuttavia possiamo intravedere una materna sollecitudine del Santo, la sua lealtà verso il frate bisognoso e la prontezza ad aiutarlo nei momenti difficili. Frate Francesco scrive al frate Leone:

Frate Leone, frate Francesco tuo (dice) salute e pace. Così ti dico, figlio mio, come una madre, che tutte le parole che dicemmo in cammino, brevemente in questa frase (te le) riassumo e consiglio, e (perciò) non è necessario che tu venga da me per (avere il mio) consiglio, poiché così io ti dico: in qualunque modo ti sembra meglio di piacere al Signore Dio e di seguirne le orme e la povertà, fate (così), con la benedizione del Signore Dio e l'obbedienza a me. E se ti è necessario [...] per (avere) ulteriore consolazione e vuoi tornare da me, vie(ni) ${ }^{56}$.

Tommaso da Celano menziona nella sua biografia, che frate Leone durante il soggiorno del Santo sul Monte La Verna nel mese di settembre 1224 ha sperimentato una grave tentazione - «non della carne ma dello spirito». san Francesco non è rimasto indifferente in una tale situazione. Ispirato dallo Spirito Santo, ha riconosciuto il desiderio di frate Leone, che, soffrendo per causa della forte tentazione, desiderava ricevere dal Santo un biglietto con l'autografo di Francesco ${ }^{57}$. Tuttavia aveva paura di rivelarlo esplicitamente a Francesco. Ma questi, appena abbia riconosciuto lo stato dello spirito di frate Leone - come lo attestano le prime biografie del Santo - lo ha chiamato dicendo: «Portami carta e calamaio, perché voglio scrivere le parole e le lodi del Signore, come le ho meditate nel mio cuore $\aleph^{58}$. Frate Leone portò ciò che chiedeva, e il Santo di proprio pugno ha scritto le lodi di Dio e le parole che desiderava. Si è rivelato ancora più generoso, perché ha scritto una benedizione speciale per frate Leone $\mathrm{e}^{59}$.

Dopo questo evento, la tentazione è scomparsa. L'esempio di questo "biglietto" offerto a frate Leone ci fa vedere molto bene in Francesco, accanto al dono di penetrazione della coscienza, anche la sua bontà e la cura verso il fratello. Riassumendo possiamo affermare che nell'atteggiamento

56 Fonti Francescane, Lettera a frate Leone 1-4.

57 Cfr. Block W., Frate Leone, “pecorella di Dio”, „Frate Francesco” 77 (2011), 7-32.

58 Tommaso da Celano, Memoriale nel desiderio dell'anima. (Vita seconda) 49.

59 Questo biglietto è una preziosissima reliquia con l'autografo di San Francesco, custodito in un costoso reliquiario nel Sacro Convento ad Assisi. Sull'una pagina Francesco ha scritto la preghiera Lodi di Dio altissimo, sull'altra la Benedizione a frate Leone. 
di san Francesco verso i frati, e soprattutto verso gli afflitti, si può scoprire un immenso amore del Santo verso di loro. Questo amore si esprime nella sensibilità a ciò che stanno vivendo, in una materna preoccupazione e delicatezza e nel rispetto verso di loro.

\subsubsection{La compassione verso i frati malati}

I frati malati erano particolarmente cari a Francesco. Lo prova il fatto che egli nomina il modo di comportarsi con loro, sottolineando i privilegi speciali per loro nel documento legislativo più importante - nella Regola. Sia nella versione non aprovata che in quella bollata troviamo evidenti, analogiche e menzioni riguardanti il modo di comportarsi verso i frati malati. Due di esse direttamente incitano a curargli, la terza invece tratta il tema dei privilegi e facilitazioni per i frati malati. Adesso, prima di considerare le prescrizioni legislative precise, contenute nelle regole, che riguardano il modo di frequentare i frati malati, l'oggetto della nostra analisi facciamo l'Ammonizione Ventiquarantesima, la più breve indicazione fra tutte lasciate da Francesco ai suoi frati. La regola del comportamento contenuta in essa diventerà la chiave per poter capire l'atteggiamento della compassione che Francesco manifestava verso i frati malati.

Nell'Ammonizione Ventiquarantesima frate Francesco ammonisce: «Beato il servo che ama il suo fratello quand'è malato e non può far niente per lui, così come quando è sano e può soddisfarlo» ${ }^{60}$. L'ammonizione s'intitola "il vero amore". L'amore autentico appare prima di tutto quale disinteressato e libero e solo in questo modo diventa il riflesso del amore vero di Gesù ${ }^{61}$. Si ama e si sente compassione facilmente verso uno da chi si può aspettare la gratitudine e ricompensa. Invece, è molto più difficile amare un ammalato che non è in grado di rendere il servizio in contraccambio. Tuttavia, Francesco incita proprio a un tale amore. Lui non vuole che il motivo di curare il frate malato sia un personale tornaconto in vista della sua guarigione. L'unica ragione della cura per il ma-

\footnotetext{
60 Fonti Francescane, Ammonizioni XXIV,1.

${ }_{61}$ Cfr. Spogli E., Temi di Pastorale Sanitaria. Conversazioni svolte a Medici, Infermieri, Cappellani Ospedalieri, Religiose Infermiere, Tinari, Villamagna 2001, p. 121.
} 
lato deve essere l'amore gratuito. Soltanto in questa situazione del servizio al frate ammalato e debole, ciascuno può rendersi conto dei veri motivi del proprio agire nascosti nel cuore. Francesco fa il riferimento all'amore verso li frate sano perché ogni frate che assiste un malato possa paragonare il suo comportamento verso un frate sano, che lo può aiutare con quello verso l'ammalato che non ha niente da offrire in ricompensa. Il messaggio fondamentale che deriva di quest'ammonizione dice: non ci può essere un amore autentico rivelandosi nelle sfumature più svariate senza un evidente tratto di disinteresse. Nella citata ammonizione, il Poverello fa appello alle più tenere sensazioni dello spirito, alle quali appartiene fra l'altro l'atteggiamento dell'amore fraterno compassionevole ${ }^{62}$.

D’altra parte, le prescrizioni precise riguardanti la cura dei frati malati sono contenute in tutte e due Regole: più diffusamente in Regola non bollata, invece, più concisamente in quella bollata. Nel capitolo sesto della Regola bollata san Francesco incita i suoi frati a una cura dei malati:

E ovunque sono e si troveranno i fratelli, si mostrino familiari a vicenda tra di loro. E senza timore ognuno manifesti all'altro le proprie necessità, perché, se la madre nutre ed ama il proprio figlio carnale, con quanto più amore deve uno amare e nutrire il suo fratello spirituale? E se uno di loro cadrà malato, gli altri fratelli devono servirlo, così come essi stessi vorrebbero essere serviti ${ }^{63}$.

Questa disposizione, Francesco la scrive nella Regola non bollata, perché il modo di vivere dei frati in quel tempo stava nel percorrere a piedi, in piccoli gruppi, i paesi e predicare. Il Santo desiderava assicurare l'assistenza ai frati che, durante questi viaggi, si sono ammalati e non potevano trovare un aiuto competente reso dai organismi convenienti ${ }^{64}$.

62 Cfr. Temperini L., Amore di Dio, amore del prossimo, [in:] Caroli E. (a cura di), Dizionario francescano, EMP, Padova 1995, p. 87; Messa P., Profili L., Il Cantico della fraternità...

63 Fonti Francescane, Regola bollata VI, 7-9.

64 In tempi di Francesco i monaci degli antichi ordini, abitualmente chiusi nei loro monasteri, venivano curati dai "monaci infermieri", in apposite sale, le infirmerei del monastero. Invece, la vita peregrinante dei frati minori nella loro estrema povertà, non aveva nulla di tutto questo. Le prime comunità francescane, soprattutto nei primi anni, non avevano conventi in muratura e quando ne cominciarono a costruire qualcuno, erano talmente piccoli e poveri, che era impossibile attrezzarvi un'infermeria. 
Un cenno analogo nella Regola non bollata rivela nettamente questa intenzione di Francesco che nella comunità alla quale appartiene il malato ci sia almeno uno che prende cura di lui personalmente:

Se qualcuno dei fratelli cadrà malato, dovunque si trovi, gli altri fratelli non lo lasciano, se non sia stato prima designato uno dei fratelli o più d'uno, se sarà necessario, che lo servano, così come essi stessi vorrebbero essere serviti. Ma in caso di estrema necessità, possono lasciarlo a una persona che debba soddisfare alla sua infermità ${ }^{65}$.

Francesco richiede che, nel caso della malattia di un frate, gli altri indichino uno che debba rimanere con l'ammalato e prenderne cura. Francesco non vuole che il frate malato sia curato da qualcuno estero alla fraternità. Una tale situazione potrebbe succedere solo nell'estrema necessità. Nelle circostanze normali i confratelli sono obbligati a occuparsi del frate bisognoso. Una preoccupazione stupenda del Santo per il frate malato si vede ancora più evidentemente nel suo ordine secondo il quale alcuni frati dovevano restare con l'ammalato in caso della necessità. Non è dunque l'attività apostolica come quella più importante per Francesco, ma quella di farsi carico di un frate malato o in bisogno ${ }^{66}$. Quando è necessario, persino più frati, invece di andare a predicare, dovrebbero rimanere con il confratello malato e assisterlo. Il Santo propone anche il modo in cui si devono comportare, perché: «lo servano, così come essi stessi vorrebbero essere serviti». Si nota qui una netta analogia al "principio d'oro di comportarsi" indicato da Gesù Cristo nel Sermone sul Monte: "Tutto quanto volete che gli uomini faccia-

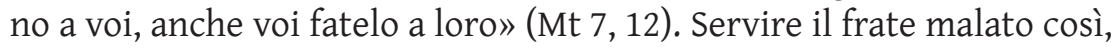
come quello che serve vorrebbe che lo servano, riporta anche al comandamento dell'amore del prossimo: «Amerai il prossimo tuo come te stesso» (Mt 22, 39; Dt 6, 5). Dunque, questo deve essere un servizio pieno d'impegno, di amore e di compassione.

L'incarico della preoccupazione per le necessità dei malati è imposto da san Francesco prima di tutto ai superiori. Loro, per così dire, d'ufficio

65 Fonti Francescane, Regola non bollata X, 1-2.

${ }_{66}$ Schmucki O., Infermità, [in:] Caroli E. (a cura di), Dizionario francescano, EMP, Padova 1995, p. 755 . 
sono obbligati di avere cura delle loro necessità. Nella Regola bollata Francesco scrive:

Tuttavia, per le necessità degli infermi e per vestire gli altri fratelli, i ministri soltanto e i custodi, ed essi soltanto, per mezzo di amici spirituali, provvedano con sollecitudine secondo i luoghi e i tempi e le fredde regioni, come sembrerà convenire alla necessità; fatto sempre salvo che, come é stato detto, non accettino monete o denaro ${ }^{67}$.

Il Santo lo dice nel contesto di ricevere il denaro e gli oggetti di valore. Per questa ragione solo i guardiani possono chiedere agli amici spirituali l'assistenza materiale per i frati malati. Tuttavia, non è permesso loro ricevere direttamente il denaro oppure gli oggetti di valore, né personalmente, da soli, disporre di essi per soddisfare ai bisogni dei malati. Sono soltanto obbligati a rivolgersi agli amici e questi devono fornire ciò che è necessario ai bisognosi. E' notevole la libertà che Francesco lascia ai superiori. Mettendo così tanto in rilievo la povertà, nonostante tutta la determinazione e il tono risoluto in riferimento al denaro, qui Francesco lascia un ampio spazio di libertà ai guardiani. Ordina loro apertamente che prendano cura dei bisogni dei farti. Sono obbligati a farlo con una maggiore premura.

Un altro cenno sui frati malati nella Regola parla dei privilegi e facilitazioni per loro. Francesco proibiva categoricamente ai frati andare a cavallo, perché allora andare a cavallo era un privilegio dei ricchi e regnanti. I frati minori invece dovevano spostarsi in modo consueto per la gente comune d'allora, cioè a piedi. Nel capitolo terzo della Regola non bollata scrive: «E non vadano a cavallo, se non costretti da manifesta necessità o da infermità» ${ }^{68}$. Unicamente una occorrenza evidente o la malattia dispensava i frati da questa legge. Per Francesco la cosa più fondamentale non è la povertà, ma l'amore per i frati nella situazione dell'estremo bisogno, cioè dell'infermità. La compassione che nasce dall'amore, abolisce ogni regola. I brani degli Scritti del Poverello da noi esaminati, concernenti la cura verso i frati malati, in modo evidente rendono lo spirito della fraternità francescana primitiva. La sensibilità e attenzione verso il frate infermo,

67 Fonti Francescane, Regola bollata IV, 2.

68 Fonti Francescane, Regola bollata III, 12. 
l'assistenza premurosa della fraternità accanto al malato, si rivelavano il criterio dell'autentica vocazione alla fraternità dei frati minori ${ }^{69}$.

\subsubsection{La compassione verso i frati peccatori}

A questo punto vogliamo vedere l'atteggiamento di Francesco verso i frati che hanno peccato. Il più fermo appello, proveniente dalla consapevolezza dello stato di peccato dell'uomo, fu per Francesco quello della necessità di amare ogni peccatore ${ }^{70}$. Esaminando gli Scritti del Santo possiamo accorgerci che lui si rivolge ai frati peccatori "che chiedono misericordia", ma anche i frati peccatori "che non chiedono misericordia". Questo sguardo di Francesco sul peccatore è uno sguardo del "padre buono" che in un modo specifico prende una posizione verso al peccatore contrito ("chiedendo perdono"), e tutt'altra posizione verso un peccatore impenitente (,che non chiede perdono”). E' una distinzione presentata dal Santo stesso nella Lettera ad un Ministro ${ }^{71}$.

Con facilità si adotta verso un peccatore l'atteggiamento di disapprovazione, indignazione o indifferenza. Ciò risulta dall'identificazione della azione cattiva con l'uomo che l'ha commessa. Francesco invece, impregnato dallo spirito della tradizione cattolica, notava una differenza sostanziale tra il peccatore e il peccato, per questo ha potuto scrivere nell'ammonizione:

Al servo di Dio nessuna cosa deve dispiacere tranne il peccato. E in qualsiasi modo qualche individuo pecchi, e per questo il servo di Dio, non a motivo di carità, si turbi e si adiri, tesaurizza per sé la colpa ${ }^{72}$.

Francesco era un uomo della fede matura, che condanna il peccato in quanto male, quale il rifiuto di Dio e una contrapposizione a Lui, ma nello stesso tempo, rispetta la persona stessa, anche se peccatore, quale la creatura più preziosa di Dio, per la quale Egli ha dato Suo Figlio ${ }^{73}$.

\footnotetext{
69 Accrocca F., Franciszek bratem i nauczycielem, Kraków 2006, pp. 120-121.

Cfr. Block W., Teologia spirituale..., p. 94.

Fonti Francescane, Lettera a un ministro 9-10.

Fonti Francescane, Ammonizioni XI, 1-2.

Cfr. Catechismo della Chiesa Cattolica, 386-388.
} 
Francesco esclude la posizione di indifferenza verso il peccato. Il peccato non può essere gradito al servo di Dio, dunque al cristiano, a maggior ragione al religioso. Il peccato deve suscitare da lui l'inquietudine, anzi l'ira, ma l'unico motivo di un tale atteggiamento deve essere l'amore verso il peccatore.

Francesco desidera, che non si rimanga indifferente davanti a tali frati, ma si affligga per causa del peccato che loro commettono. Invoca che non si offenda né rattrista per l'ingiustizia provata, ma gli presentare l'amore attivo. Dunque, lui ama ogni peccatore, e soprattutto il frate che commette il peccato. Questo sguardo pieno di compassione, il Santo lo attingeva dalla consapevolezza del fatto che ogni uomo redento da Gesù Cristo diventa figlio di Dio, che solo ha diritto di giudicare l'uomo. Francesco, fissando lo sguardo su Gesù, cercava di imitarLo secondo le parole pronunciate da Gesù stesso: «Non sono i sani che hanno bisogno del medico, ma i malati; io non sono venuto a chiamare i giusti, ma i peccatori a convertirsi» (Lc 5, 31-32).

L'esigenza successiva che il Santo poneva davanti ai superiori è la dominazione sull'ira e sulla collera verso i frati peccatori. Francesco, se mette le tale prescrizioni nelle regole, doveva conoscere diverse reazioni dei ministri ai peccati dei frati. L'atteggiamento giusto del superiore verso il frate che ha peccato, ma che chiede il perdono, deve essere serenità e benignità. Il superiore è obbligato a soccorrere spiritualmente chi ha peccato, e non arrabbiarsi con lui e aggiungere al suo peccato anche il proprio ${ }^{74}$. La collera e l'irritazione sono contrarie all'amore che in questo caso si manifesta nella pazienza e umiltà.

Francesco, nella Lettera ad un Ministro, diceva che vuole introdurre nella regola il capitolo che trattasse sul comportarsi con i frati che commettevano un peccato gravissimo. Il desiderio di Francesco è che tutti i frati - non solo i guardiani - non facciano vergogna né diffamino il frate che era caduto, ma che tutti gli mostrino misericordia. «E tutti i frati, che sapessero che egli ha peccato, non gli facciano vergogna né dicano

${ }^{74}$ Cfr. Fonti Francescane, Regola non bollata V, 7-8; Fonti Francescane, Lettera ai fedeli (recensio brevis) $42-44$. 
male di lui, ma ne abbiano grande misericordia e tengano molto privato il peccato del loro fratello...» $\rangle^{75}$.

L'amore vero verso Cristo si deve mostrare nell'atteggiamento di una misericordia incondizionata. Il Santo raccomanda che, anche se un frate avesse peccato più gravemente e si rivolgesse al ministro chiedendo perdono, colui deve perdonarlo. Nella stessa lettera Francesco scrive ad un ministro:

E in questo voglio conoscere se tu ami il Signore e me, servo suo e tuo, se tu farai così, ovvero che non vi sia alcun fratello al mondo, che abbia peccato, quanto è possibile peccare, che, dopo aver visto i tuoi occhi, mai se ne vada senza la tua misericordia, qualora abbia chiesto misericordia ${ }^{76}$.

Francesco scorgeva il pericolo non solo nel peccato, ma anche nella punizione non adatta e nel trattamento inopportuno del superiore verso li frate peccatore. Perciò avvertisse i ministri:

E badino tutti i fratelli, tanto i ministri servi quanto gli altri, di non turbarsi o adirarsi a causa del peccato o del cattivo esempio altrui, perché il diavolo per la colpa di uno solo vuole corromperne molti. Invece spiritualmente, come meglio possono, aiutino colui che ha peccato ${ }^{77}$.

Francesco sapeva in un modo incomparabile unire fermezza e inflessibilità nel riprendere e punire con una immensità di misericordia e di clemenza. Sapeva quando e perché punire, e quando mostrare misericordia. Metteva però in rilievo e raccomandava piuttosto quell'ultimo atteggiamento. Come esempio possiamo citare la Lettera ad un ministro, menzionata qui sopra. Le due frasi indirizzate al ministro sono più significative per descrivere l'atteggiamento del Santo di cui parliamo:

E se non chiedesse misericordia, tu chiedi a lui, se vuole misericordia. E se mille volte peccasse davanti ai tuoi occhi, amalo più di me per questo: affinché tu lo tragga al Signore; ed abbi sempre misericordia per tali fratelli ${ }^{78}$.

\footnotetext{
75 Fonti Francescane, Lettera a un ministro 15.

76 Fonti Francescane, Lettera a un ministro 9.

77 Fonti Francescane, Regola non bollata V, 7-8.

78 Fonti Francescane, Lettera a un ministro 10-11.
} 
Anche la ripetizione del peccato non dispensa il ministro nel mostrare la misericordia: «E se mille volte peccasse davanti ai tuoi occhi, amalo più di me». Se il frate che ha ricevuto il perdono, commettesse ancora una volta il peccato, anche sotto gli occhi del ministro, questi deve mostrargli misericordia. Il superiore deve comportarsi così senza riguardo al fatto, quante volte il frate commette il peccato - persino "mille volte», cioè sempre. Si vede qui il riferimento chiaro alla risposta di Gesù data a Pietro quando costui chiedeva: «Allora Pietro gli si avvicinò e gli disse: "Signore, quante volte dovrò perdonare al mio fratello, se pecca contro di me? Fino a sette volte?". E Gesù gli rispose: "Non ti dico fino a sette, ma fino a settanta volte sette"» (Mt 18, 21-22).

Le considerazioni effettuate ci portano dunque a concludere che il Santo nelle relazioni con i frati che commettono il peccato pone l'accento sulla misericordia e sul perdono. Parla anche dell'ammonimento e del punire questi frati. Ritiene che li perdono e il mostrare misericordia con pazienza sia più efficace che punire i frati che si sono degradati. Lo riferisce ai frati "che chiedono perdono" come a quelli "che non chiedono perdono". Basandosi sui frammenti dei testi presi dagli Scritti del Santo si vede chiaramente non sono l'attenzione e premura di Francesco nei confronti dei frati peccatori, ma anche la sua solidarietà espressa nel amore misericordioso. Ogni frate che soffriva spiritualmente gli era caro. Questo atteggiamento si nota in particolar modo nella Lettera ad un Ministro, che tra gli altri scritti del Santo rappresenta la più bella prova della sua attenzione e delicatezza sia verso i frati peccatori, sia verso quelli afflitti.

\subsection{La compassione verso i nemici}

Nell'ambito della compassione manifestata da san Francisco agli altri non ha potuto mancare il posto per i nemici. L'amore per i nemici costituisce una specie sommamente nobile e, nel contempo, la più difficile dell'amore reciproco. L'attuazione del commando di Gesù: «amate i vostri nemici e pregate per i vostri persecutori» (Mt 5, 44), diventa la misura di una fede matura e di un amore perfetto. Nel capitolo ven- 
tidue della Regla non bollata troviamo la caratteristica del nemico inteso da Francesco:

\begin{abstract}
Presentiamo attenzione, fratelli tutti, a ciò che dice il Signore: Amate i nostri amici e fate del bene a quelli che vi odiano. Infatti anche il Signore nostro Gesù Cristo, del quale dobbiamo seguire le orme, chiamò amico il suo traditore si offrì spontaneamente ai suoi crocifissori. Sono pertanto amici nostri tutti quelli che ingiustamente ci infliggono tribolazioni e sofferenze, umiliazioni e offese, dolori e tormenti, martirio e morte. Dobbiamo molto amare costoro, poiché per quello che ci infliggono abbiamo la vita eterna ${ }^{79}$.
\end{abstract}

Il Santo nel suo ragionamento si richiama a una semplice argomentazione: chi, attraverso la sua condotta, ci costringe a oltrepassare i limiti del nostro egoismo e della nostra superbia, in realtà ci aiuta ad osservare meglio il Vangelo, e con ciò diventare simile a Cristo. Una tale persona non può essere considerata nemica, perché, presentandosi sul nostro sentiero, in effetti contribuisce alla nostra trasformazione e santificazione ${ }^{80}$. In questo senso perfino i nemici diventano amici. Il criterio di un tale atteggiamento è il principio al quale invita san Francesco nell'Ammonizione novantesimo:

Dice il Signore: Amate i vostri nemici eccetera. Ama infatti veramente il suo nemico colui che non si duole per l'ingiuria che gli fa, ma a causa dell'amore di Dio brucia per il peccato della sua anima. E nell'agire gli dimostri amore ${ }^{81}$.

La fonte dell'amore compassionevole e della pazienza nel sopportare le avversità da parte dei nemici dovrebbe diventare la sollecitudine per il loro bene spirituale, che nasce dall'esperienza dell'amore incondizionato di Dio. L'espressione di questa attenzione non può essere solamente la preghiera che diventa lo scopo in sé, ma la preghiera che genera l'amore. Secondo il Poverello, l'amore deve esprimersi in una azione concreta. In questo modo si rivela una dinamica dell'amore attivo che rende l'uomo simile a Gesù. Il processo intero si iscrive nella vita animata dal messaggio delle Beatitudini al quale deve tendere la vita cristia-

\footnotetext{
79 Fonti Francescane, Regola non bollata XXII 1-4.

80 Cfr. Synowczyk K., Miłość w nauczaniu..., p. 73.

81 Fonti Francescane, Ammonizioni IX 1-4.
} 
na: «Beati voi quando vi insulteranno, vi perseguiteranno e, mentendo, diranno ogni sorta di male contro di voi per causa mia. Rallegratevi ed esultate, perché grande è la vostra ricompensa nei cieli» (Mt 5, 11-12).

Negli Scritti del Santo troveremo ancora un altro motivo riferendosi all'amore dei nemici, quale è l'amore misericordioso manifestato nell'atteggiamento del perdono. Nel Commento al Padre Nostro, commentando la richiesta della remissione dei peccati, Francesco scrive:

Come noi li rimettiamo ai nostri debitori: e quel che non rimettiamo pienamente, tu, o Signore, fa' che lo rimettiamo pienamente, cosicché amiamo veracemente i nemici per te, e intercediamo devotamente per loro presso di te, non rendendo ad alcuno male per male e in te ci studiamo di giovare i ogni $\cos ^{82}$.

Francesco sa molto bene che è difficile perdonare chi ci ha offeso, molte volte si è incapaci. E cosciente che soltanto Lui, Signore, può darci la forza di farlo: «tu, o Signore, fa' che lo rimettiamo pienamente». Il perdono è l'espressione più profonda dell'amore fraterno ed è il contrassegno e la verifica della carità. Amici o nemici, ladri e briganti tutti devono di essere "oggetto" di compassione e di amore ${ }^{83}$. Il perdono diventa così l'evento fondamentale che trasforma il cuore e la vita di ognuno portandolo ad amare tutti con la bontà di Padre celeste: «L'amore del Padre e la passione del Figlio sono le prime e fondamentali cause che ci donano il perdono dei peccati $»^{84}$. La consapevolezza della remissione dei propri peccati, nel momento in cui, tramite il peccato noi stessi ci facciamo nemici di Dio, può generare in noi un legame affettuoso che deriva dalla compassione di fronte alla sorte dei nostri nemici ${ }^{85}$. Per questa ragione Cristo ha collegato la remissione dei nostri peccati con la condizione che anche noi dobbiamo rimettere ai nostri debitori. Un tale comportamento, essendo l'imitazione dell'amore di Dio che perdona, comprende la somma misura dell'amore del prossimo ${ }^{86}$.

\footnotetext{
82 Fonti Francescane, Preghiera sul Padre Nostro 8.

83 Fonti Francescane, Regola non bollata VII, 15.

84 Lehmann L., Francesco maestro di preghiera..., p. 215.

85 Cfr. Rm 5, 8-10.

${ }^{86}$ Cfr. Lehmann L., Francesco maestro di preghiera..., p. 213-214.
} 


\section{La compassione per tutta la Creatura}

Francesco ha scoperto nella creazione un «simbolo» di Dio, dove l'uomo occupa un posto particolare in quanto sua «immagine e somiglianza». Dunque, la concezione della creazione è chiaramente vista in modo antropocentrico ${ }^{87}$. Negli Scritti, il tema della creazione non ha un ruolo principale. Francesco svolge le sue riflessioni prima di tutto intorno alla relazione tra Dio e l'uomo, meno invece intorno alle relazioni tra l'uomo e le altre creature. Tuttavia, possiamo trovare nei suoi pochi testi il riferimento alla relazione tra l'uomo e le creature.

L'identificazione con Gesù Cristo povero e sofferente è una costante nel pensiero di Francesco ${ }^{88}$. L'esempio di Gesù che passò la sua vita terrena nell'umiltà dell'Incarnazione e nell'umiliazione della Croce ha causato che frate Francesco sentisse il desiderio di farsi frate minore come frate di tutti e di tutto ${ }^{89}$. In Cristo, Francesco si riconosce il primogenito di tutta la creazione, cioè fratello di tutti. In questa prospettiva, la relazione tra l'uomo e il creato assume una nuova valenza che deriva non solo dalla comune paternità in Dio come Padre, ma anche dalla comune fratellanza in Cristo: «...dallo stesso atto creativo nasce una co-figliolanza rispetto al Dio padre e una co-fratellanza rispetto al Dio - Figlio incarnato in Gesù Cristo» ${ }^{90}$. Nell'atteggiamento di minorità si esprime la relazione con l'altro che crea la vicinanza con gli uomini ma anche rivela la nuova relazione con tutto l'ambiente ${ }^{91}$.

Un aspetto particolare della vita di san Francesco emerge dalla minorità è la fratellanza, mediante le quali egli esprime il suo amore e la compassione nei confronti della creatura. Negli Scritti del Santo troviamo assai significativa la sua opera scritta nel periodo in cui è diventato quasi com-

${ }^{87}$ Cfr. Freyer J. B., “Homo Viator”..., p. 111.

88 Cfr. Cozzaglio P., La sincronicità nel cammino spirituale e mistico di san Francesco d'Assisi, „L'Italia Francescana” 3 (2001), p. 34.

${ }^{89}$ Il minores era la categoria sociale nel Medioevo che raccoglieva le persone più povere e senza nome nella società in contrario l'altra categoria che erano i maiores, persone agiate e borghesi.

${ }_{90}$ Freyer J. B., “Homo Viator”..., pp. 112-113.

91 Cfr. Vaiani C., Per una lettura..., pp. 90-91. 
pletamente cieco $^{92}$. Si tratta di una poesia religiosa di altissimo livello che conferma e rivela il suo atteggiamento di fratellanza, cioè il Cantico di frate Sole, chiamato anche Cantico delle Creature:

\begin{abstract}
Altissimo, onnipotente, bon Signore, tue so le laude, la gloria e l'onore et onne benedizione. A te solo, Altissimo, se confano e nullo omo è digno te mentovare. Laudato si, mi Signore, cun tutte le tue creature, spezialmente messer lo frate Sole, lo qual è iorno, e allumini noi per loi. Ed ello è bello e radiante cun grande splendore: de te, Altissimo, porta significazione. Laudato si, mi Signore, per sora Luna e le Stelle: in cielo l'hai formate clarite e preziose e belle. Laudato si, mi Signore, per frate Vento, e per Aere e Nubilo e Sereno e onne tempo per lo quale a le tue creature dai sustentamento. Laudato si, mi Signore, per sor Aqua, la quale è molto utile e umile e preziosa e casta. Laudato si, mi Signore, per frate Foco, per lo quale enn'allumini la nocte: ed ello è bello e iocundo e robustoso e forte. Laudato si, mi Signore, per sora nostra matre Terra, la quale ne sostenta e governa, e produce diversi fructi con coloriti flori ed erba. Laudato si, mi Signore, per quelli che perdonano per lo tuo amore e sostengo infirmitate e tribulazione. Beati quelli che 'l sosterrano in pace, ca da te, Altissimo, sirano incoronati. Laudato si, mi Signore, per sora nostra Morte corporale, da la quale nullo omo vivente po' scampare. Guai a quelli che morrano ne le peccata mortali! Beati quelli che trovarà ne le tue santissime voluntati, ca la morte seconda no li farrà male. Laudate e benedicite mi Signore, e rengraziate e serviteli cun grande umilitate ${ }^{93}$.
\end{abstract}

Il Cantico di frate Sole è senza dubbio la più celebre opera del Santo. Quando si parla di Francesco d'Assisi, non si può non andare con la mente a questo cantico che manifesta appieno la sua dimensione umano - spirituale e, particolarmente, la sua vena poetica, sebbene va pur considerato come venga sottolineato più l'aspetto letterario che quello mistico - religioso. Esso è un canto mistico, nato da un profondo e vissuto rapporto con Gesù Cristo, che nel Santo d'Assisi si rese visibile anche esternamente attraverso l'esperienza delle stigmate $^{94}$. Inoltre, il Cantico di frate Sole, che appartiene alla letteratura mondiale, secondo Ernesto Renan potrebbe essere considerato «il più bel brano di poesia religiosa dopo il Vangelo» ${ }^{95}$.

92 Cfr. Cargnioni C., Malato nella visione di s. Francesco, [in:] Horowski A. (a cura di), Religioni et doctrinae. Miscellanea di studi offerri a Bernardino de Armellada in occasione del suo $80^{\circ}$ compleanno, Roma 2009, p. 57.

93 Fonti Francescane, Cantico di frate Sole 1-33.

94 Cfr. Lehmann L., Francesco maestro di preghiera..., p. 345.

95 Cfr. Ibidem, p. 328. 
Secondo la maggioranza degli studiosi il cantico - composto tra il 1225 e il 1226 - è stato redatto in tre momenti diversi: la prima parte durante il soggiorno a San Damiano; la seconda, la strofa del perdono, quando ad Assisi scoppia il dissidio tra il vescovo ed il podestà; la terza, infine, quella sulla morte, prima di morire. Sulla sua autenticità non ci sono dubbi; esso infatti è riportato dal manoscritto d'Assisi, il famoso codice 338 scritto intorno al 1250. In questo manoscritto il testo viene preceduto da un'introduzione dove si legge: «Qui inizia il canto di lode delle creature composto dal beato Francesco a lode e gloria di Dio quando era malato in San Damiano ${ }^{96}$. Un'altra testimonianza che accredita l'autenticità del cantico proviene da Tommaso da Celano - suo discepolo e primo biografo - il quale, due anni dopo morte del Santo, nella sua Vita prima crea una connessione e paragona il Cantico di frate Sole con il Canto dei tre fanciulli nella fornace ardente che troviamo nell'Antico Testamento (Dan 3, 57-90) ${ }^{97}$.

Da quando Francesco ha incontrato Dio, le creature gli si rivelano con un volto nuovo, e diventano il mezzo privilegiato per arrivare a Dio stesso. Nella modalità con la quale Francesco racconta la sua particolare esperienza di Dio tramite il Cantico di frate Sole possiamo intravedere il volto di Dio compassionevole ${ }^{98}$. Francesco, loda e apprezza le creature anche in un contesto normalmente non accettabile, cioè quello di sofferenza. Il Cantico delle creature è l'opposto di un invito al disprezzo delle realtà visibili; esso infatti guida il nostro sguardo verso l'ordine e la bellezza della creazione materiale, cosicché anche ciò che nell'esistenza appare assurdo e penoso - la sofferenza e la morte - viene trasfigurato. Tuttavia il Cantico di frate Sole non è solo e semplicemente una lode del creato, bensì anche una dossologia rivolta all'«Altissimu, onnipotente bon Signore» che «nullu homo è dignu ... mentovare». A lui solo è rivolta la lode, per e attraver-

96 Cfr. Ibidem, p. 331.

97 Tommaso da Celano, Vita del beato Francesco. (Vita prima) 80: „Come un tempo i tre fanciulli gettati nella fornace ardente invitavano tutti gli elementi a glorificare e benedire il Creatore dell'universo, così quest'uomo, ripieno dello spirito di Dio, non si stancava mai di glorificare, lodare e benedire, in tutti gli elementi e in tutte le creature, il Creatore e governatore di tutte le cose".

${ }_{98}$ Kruszyńska A., Wizjaświata w “Pochwale stworzenia” św. Franciszka, „Studia Franciszkańskie” 15 (2005), p. 109. 
so tutte le creature, che manifestano qualcosa della sua gloria splendente. Francesco ha composto questo canto mentre, gravemente ammalato e quasi cieco, attraversava una notte dello spirito: uno sguardo reso puro dalla sofferenza e dalle lacrime si rivolge al mondo ${ }^{99}$.

Dopo le strofe che si soffermano meravigliate sulla bellezza della creazione, il testo ci conduce all'uomo: non l'uomo nella sua bellezza e forza, ma quello ferito dall'offesa, segnato dalla malattia e dall'angoscia, consegnato agli artigli della morte. Di fronte all'armonia delle creature, in opposizione rispetto ad essa, si apre un mondo di negatività: la sofferenza umana e il suo inevitabile compimento, la morte. E tuttavia, anche per e attraverso questa realtà «laudato si', mi' Signore», poiché l'uomo, grazie all'amore che Dio gli ha rivelato, può perdonare e sopportare nella pace malattia e sofferenza ${ }^{100}$.

Per il nostro tema è interessante notare una geniale intuizione che permette a Francesco di svelare una specie di parentela di sangue con gli esseri inanimati: i sostantivi "fratello" e "sorella", oltre che alludere all'identica famiglia, ispirano anche una certa tenerezza. Max Scheler occupandosi di Francesco d'Assisi nella sua opera "Essenza e forme della simpatia" afferma che ciò che immediatamente ci colpisce, nell'atteggiamento del Santo è:

il fatto ch'egli chiamasse "fratelli" e "sorelle" anche il sole e la luna, l'acqua e il fuoco, ed anche gli animali e le piante d'ogni specie - che attuasse un'espansione della mozione specificamente cristiana dell'amore di Dio come Padre, dell'amore fraterno e dell'amore del prossimo "in" Dio, a tutta la natura infraumana, e che attuasse al contempo, o sembrasse attuare, un'elevazione della natura alla luce e allo splendore del sovrannaturale ${ }^{101}$.

Questa percezione del mondo per Francesco era una grande novità; sembra infatti che egli non avesse alcun precursore e che fosse il primo in tutta la storia cristiana dell'Occidente fin anche al Medioevo. La benemerenza del Santo è senza dubbio:

99 Cfr. Matura T., Francesco, un altro volto..., p. 161; Block W., „Pieśń słoneczna”, [in:] A. J. Nowak (a cura di), Homo novus, Lublin 2002, 253-277.

100 Ibidem, p. 162.

101 Scheler M., Essenza e forme..., pp. 156-157. 
tentativo di ridurre all'unità e alla sintesi, in un solo processo di vita, la mistica dell'amore della compassione universale - mistica acosmica e personale, che non guarda più dall'alto in basso, ma bensì dal basso in alto - introdotta dal cristianesimo e fusa in un'unità insieme con l'amore di Gesù, e l'unipatia cosmovitale con l'essere e con la vita della natura ${ }^{102}$.

Questa riconciliazione con tutti gli elementi della natura è evidenziata in modo più espressivo nell'uso ricorrente dell'appellativo «fratelli» e «sorelle», qualifica che, con questa composizione, viene impiegata per la prima volta ed esplicitamente nella letteratura cristiana e che, in rapporto al Nuovo Testamento, costituisce un indubbio ampliamento del concetto di fratello. Ogni creatura acquista nella lode di Francesco un volto fraterno. Francesco mediante un atteggiamento di tenerezza e riverenza si unisce con tutto ciò che fa parte dell'opera di Dio. Egli non si perde nel cosmo e nella moltitudine delle creature, ma, grazie alla qualifica di fratello - sorella, tutto gli è familiarmente vicino ${ }^{103}$. Alle espressioni che rivelano nel Cantico l'atteggiamento del Santo verso ogni creatura si aggiungono le testimonianze dei biografi, soprattutto quella di Tommaso da Celano, il suo primo biografo ${ }^{104}$.

Nell'ambiente del Santo non mancò certo la consapevolezza della grande singolarità, novità e del carattere inusitato del suo atteggiamento. È assolutamente certo che Francesco era lontano da ogni forma di panteismo. Nella visione che Francesco ha della relazione tra l'uomo e la creazione, è chiaro come egli veda l'azione creatrice di Dio come un processo d'amore che tende a un compimento interiore ed esterio$\mathrm{re}^{105}$. Per il Poverello d'Assisi il Dio come «Padre» non si mostra esclusivamente come Padre dell'uomo, ma anche come Padre amoroso di tutta la natura, avendo infatti ogni creatura origine dall'atto creativo dello stesso Padre, «perché tu [...] hai creato tutte le cose spirituali, e corpo-

102 Ibidem, p. 156.

103 Cfr. Lehmann L., Francesco maestro..., p. 343.

104 Tommaso da Celano, Vita del beato Francesco. (Vita prima) 81: „E finalmente chiamava tutte le creature con il nome di fratello e sorella, intuendone i segreti in modo mirabile e noto a nessun altro, perché aveva conquistato la libertà della gloria riservata ai figli di Dio".

105 Cfr. Freyer J. B., “Homo Viator”..., p. 121. 
rali, e noi fatti» $»^{106}$. Questa novità nello sguardo di Francesco viene sottolineata da Max Scheler:

nel rapporto emozionale di Francesco con la natura è il fatto che i prodotti e i processi della natura hanno un senso espressivo proprio, senza una relazione allegorica con l'uomo e con le situazioni umane in generale. Che anche il sole, la luna, il vento ecc., che non hanno alcun bisogno dell'amore caritatevole e compassionevole, vengano salutati ed esperiti dall'anima come fratelli e sorelle; che le creature, anche nel loro contesto metafisico (e solo con l'inclusione dell'uomo), vengano rapportate immediatamente al loro Creatore e "Padre" come esseri esistenti per sé ed anche (in rapporto all'uomo) del tutto indipendenti nel loro valore: è questo l'elemento nuovo, sorprendente, straordinario, antiebraico, nell'atteggiamento del Santo. [...] In ogni prodotto della natura la sua anima piena d'amore vede un'opera dell'invisibile e spirituale Dio creatore, una pedana di lancio dalla natura verso Dio, uno sgabello dei a suoi piedi, una rivelazione del suo splendore - una "gloria", divenuta visibile e udibile, del Signore e Padre. In questo senso, anche per lui la natura non è un oggetto immediato dell'unipatia con una vita universale distinta da Dio od anche equiparata a Dio (come per i panteisti del Rinascimento), ma è solamente un simbolo o allegoria - come mette in luce abbastanza chiaramente il "Cantico delle creature" 107.

Uomini e creature hanno lo stesso Padre, che è Padre anche di Gesù. Francesco nella persona di Cristo si riconosce il primogenito di tutta la creazione. Come l'apostolo Paolo nel suo famoso inno cristologico della lettera ai Colossesi così anche Francesco è cosciente della posizione centrale di Gesù Cristo nell'Universo: «Egli è immagine del Dio invisibile, primogenito di tutta la creazione, poiché in lui furono create tutte le cose nei cieli e sulla terra, quelle visibili e quelle invisibili... È piaciuto infatti a Dio che abiti in lui tutta la pienezza e che per mezzo di lui e in vista di lui siano riconciliate tutte le cose...» (Col 1, 15-16; 19-20).

Francesco vive in Cristo una comunione cosmica e una partecipata unione emotiva con tutto il creato, anche con gli animali. «Tale esperienza diretta della simbolica di Cristo, che trasferisce la compassione nei confronti del Signore sofferente perfino agli animali, potrebbe forse rappresentare nella storia della spiritualità un caso unico» ${ }^{108}$. Mediante l'opera

106 Fonti Francescane, Regola non bollata 23, 1.

107 Scheler M., Essenza e forme..., pp. 158-159.

108 Schmucki O., Das Leiden Christi im Leben des hl. Franziskus von Assisi, Roma 1960, p. 62; Lehmann L., Francesco maestro..., pp. 348-349. 
di riconciliazione del Figlio prediletto, tutto è stato riunito in una fraternità cosmica. Tutte le cose sono state create dal Padre. Francesco, come fratello di tutte le creature, all'interno di una liturgia cosmica, vuole lodare e ringraziare con esse, attraverso esse e per esse il Padre eterno ${ }^{109}$.

\section{Conclusione}

Concludendo le nostre considerazioni sul tema della compassione in Francesco d'Assisi nei confronti degli altri e per il Creato - esaminando gli Scritti di san Francesco - possiamo affermare che l'esperienza dell'amore di Dio rivelatosi nella storia della vita del Santo di Assisi ha portato l'influsso determinante sulla presenza di quest'atteggiamento nel suo agire. L'immagine dell'uomo formato alla luce della Bibbia gli ha permesso scoprire una dignità e grandezza che porta in sé l'uomo essendo l'opera di Dio. Per Francesco, questa dignità proviene del fatto che non solo l'anima, ma anche il corpo dell'uomo è stato formato ad immagine di Cristo. Il corpo umano è un mezzo per la presenza del Signore tra gli uomini. La corporeità rappresenta per l'uomo un modo di essere nel mondo, di mettersi in relazione con tutte le opere dell'Altissimo.

Il punto di partenza nell'esperienza della compassione per Francesco è contemplazione dell'amore di Dio per il mondo e soprattutto per il l'uomo. La contemplazione del volto del Signore definisce la risposta umana all'amore di Dio la quale si attua, secondo san Francesco, nella compassione. La compassione in Francesco verso l'uomo e la creatura nasce dalla contemplazione di Gesù povero e umile. A partire dall'incontro con il lebbroso Francesco era sempre più vicino agli uomini soprattutto quelli sofferenti e disprezzati. La sua vicinanza mostrava non soltanto verso i suoi frati ammalati ma anche quelli peccatori. Nei suoi Scritti Francesco accenna anche ai nemici, per i quali provava compassione come una specie più alta dell'amore del prossimo.

109 Cfr. Lehmann L., Francesco maestro..., p. 349. 


\section{Streszczenie}

Studium fenomenu współczucia u św. Franciszka z Asyżu został przeprowadzony na podstawie pism świętego. Franciszek zwraca się z zachętą do współczucia innym ludziom, jak i całemu stworzeniu. Swoje przesłanie kieruje przede wszystkim do podzielających wraz z ten styl przeżywania wiary, jaki on sam otrzymał od Najwyższego, a Kościół katolicki zatwierdził w Regule. Artykuł został podzielony na cztery części (Wizja człowieka w myśli św. Franciszka, Spotkanie z trędowatymi - odkrycie fenomenu współczucia, Współczucie wobec: ludzi chorych, biednych, braci, pogardzanych, wrogów; Zachowanie Franciszka wobec innych stworzeń). Ze studium pism Franciszka z Asyżu wynika, iż podstawową jego relacji do innych ludzi i pozostałych stworzeń było przede wszystkim doświadczanie miłości Boga we własnym życiu. Doświadczenie to wpływało na zarówno na jego sposób działania, jak i na uznanie godności człowieka jako dzieła Boga. Dla Franciszka cały człowiek (dusza i ciało) jest stworzony na obraz Boży, dlatego ciało ludzkie jest traktowane przez świętego jako środek do obecności Boga pośród ludzi. Ludzka cielesność jest sposobem bycia w świecie, wchodzenia w relacje z całym stworzeniem Najwyższego Boga. Współczucie u Franciszka rodzi się z kontemplacji Jezusa ubogiego i pokornego. Stąd bliskość Franciszka z ludźmi cierpiącymi, wzgardzonymi, braćmi chorymi, grzesznikami i wrogami. Wobec tych wszystkich ludzi Franciszek doświadczał współczucia, będącego wyrazem najwyższej miłości wobec bliźniego.

Słowa kluczowe: Św. Franciszek, współczucie, człowiek, ludzie, stworzenie

\section{St. Francis' compassion for people and the other creatures}

In my studies I focused on the writings of St. Francis. St. Francis turn to us asking for compassion resulted from my analysis of his writings. St. Francis encourages us to share compassion with the others and the whole creation. He directed his message most of all to those of us who share the same feelings for faith. The faith which he was granted from God St. Francis wrote down in his Rule which was subsequently endorsed by the Catholic Church. My paper consists of four parts: The image of man in St. Francis ' writings, The meeting of the leper, The sick, the poor, the abandoned, the enemies, His attitude to the whole God's creatures. His inspiration for love St. Francis drew from his personal experience of God's love. He believed that human dignity was bestowed by god himself. Man was created by God and modeled on His own image, argued St. Francis therefore man testifies to God's presence in the world. St. Francis' compassion originated from poor and humble Jesus Christ. St. Francis' compassion with the poor, sick, suffering, despised, sinners and enemies draws inspiration from the same spiritual source.

Keywords: St. Francis, compassion, human, people, creatures 